\title{
Ciclo de Mejora en el Aula Integrado: La Enseñanza del Entorno en el Grado de Educación Infantil
}

\section{Integrated cycle of improvement in the classroom: Teaching the Environment in Early Childhood Education}

FÁTIMA RODRÍGUEZ-MARÍN

Universidad de Sevilla

Dpto. Didáctica de las Ciencias Experimentales y Sociales

frodmar@us.es

ORCID: https://orcid.org/0000-0003-0771-6944

DOI: http://dx.doi.org/10.12795/9788447231003.086

Pp.: 1855-1883 


\section{Descripción del contexto}

Desde el inicio de nuestra participación en la Red de Formación e Innovación Docente (REFID) hemos apostado por la coordinación entre asignaturas, puesto que la considerábamos necesaria para el buen desarrollo de nuestras materias. En CIMAS anteriores (Rodríguez-Marín, 2018 y Puig, 2018) hemos ido realizando de manera coordinada algunos bloques de contenidos y en esta ocasión, nos proponemos llevar a cabo un CIMA integrado que abarque por completo las asignaturas de Conocimiento del Entorno Social en Educación Infantil y Enseñanza del Entorno Natural en la Etapa de 0-6 años; ambas materias obligatorias del 3o curso del Grado en Educación Infantil.

La experiencia positiva en los CIMAS anteriores nos ha llevado a apostar por la coordinación plena, a pesar de que en este curso contamos con la dificultad añadida de las derivadas de la crisis sanitaria, que nos impedirán realizar sesiones presenciales conjuntas (ambas docentes en el aula con el grupo completo de estudiantes) y salidas a los centros educativos para aplicar lo aprendido en clase.

La coordinación docente entre materias nos ha ayudado a dar una visión diferente a nuestras asignaturas, dotándolas de más significado para el alumnado y permitiéndonos que se acerquen más a la realidad de las aulas de Educación Infantil. Con el tiempo hemos ido reformulando los bloques de contenidos para ir creando una secuencia conjunta. En lo que respecta al alumnado, hasta el momento, la respuesta a esta forma de trabajo ha sido gratificante, puesto que, aunque es cierto, que en los inicios dudan de una coordinación real, conforme avanza el proceso y entran en nuestra dinámica diaria, manifiestan sentirse a gusto con el trabajo y agradecen la manera de llevar a cabo el proceso.

Ciclos de Mejora en el Aula (2020). Experiencias de Innovación Docente de la US Esta obra se distribuye con la licencia Creative Commons 


\section{Diseño previo del CIMA}

Describimos a continuación los diferentes elementos que componían el diseño previo del ciclo de mejora:

\section{Mapa de contenidos y problemas claves}

Presentamos a continuación un mapa general que abarca los grandes problemas que se trabajan en ambas materias (Figura 1).

En la parte superior del mapa podemos encontrar el problema 1 de cada asignatura: ¿Qué aportan las ciencias al área de Conocimiento del Entorno? y ¿Cómo se hace una investigación? Ambos problemas se trabajan en paralelo, cada uno desde una de las asignaturas, pero de forma conectada, puesto que se retroalimentan. Dentro de cada uno de ellos podemos encontrarnos subproblemas que nos llevarán directamente a los contenidos propios de esta primera parte.

En la parte inferior del mapa podemos ver el otro gran problema de las dos asignaturas: ¿Son los proyectos y los rincones buenas estrategias metodológicas para la Enseñanza del Entorno en Educación Infantil?, en torno al cual se desarrollan la mayoría de los bloques de contenidos. De este modo, cada uno de los subproblemas de este bloque se corresponden casi por completo con un bloque de contenidos o unidades de las dos materias.

En el mapa hemos querido recoger los contenidos de tipo conceptual y procedimental dentro de los cuadrados que van conectados con los subproblemas y los contenidos de carácter actitudinal, que consideramos transversal a las dos materias, aparecen a modo de círculos alrededor de todo el mapa.

Ciclos de Mejora en el Aula (2020). Experiencias de Innovación Docente de la US Esta obra se distribuye con la licencia Creative Commons 


\section{MARÍA PUIG GUTIÉRREZ Y FÁTIMA ROdRÍGUEZ-MARÍN}

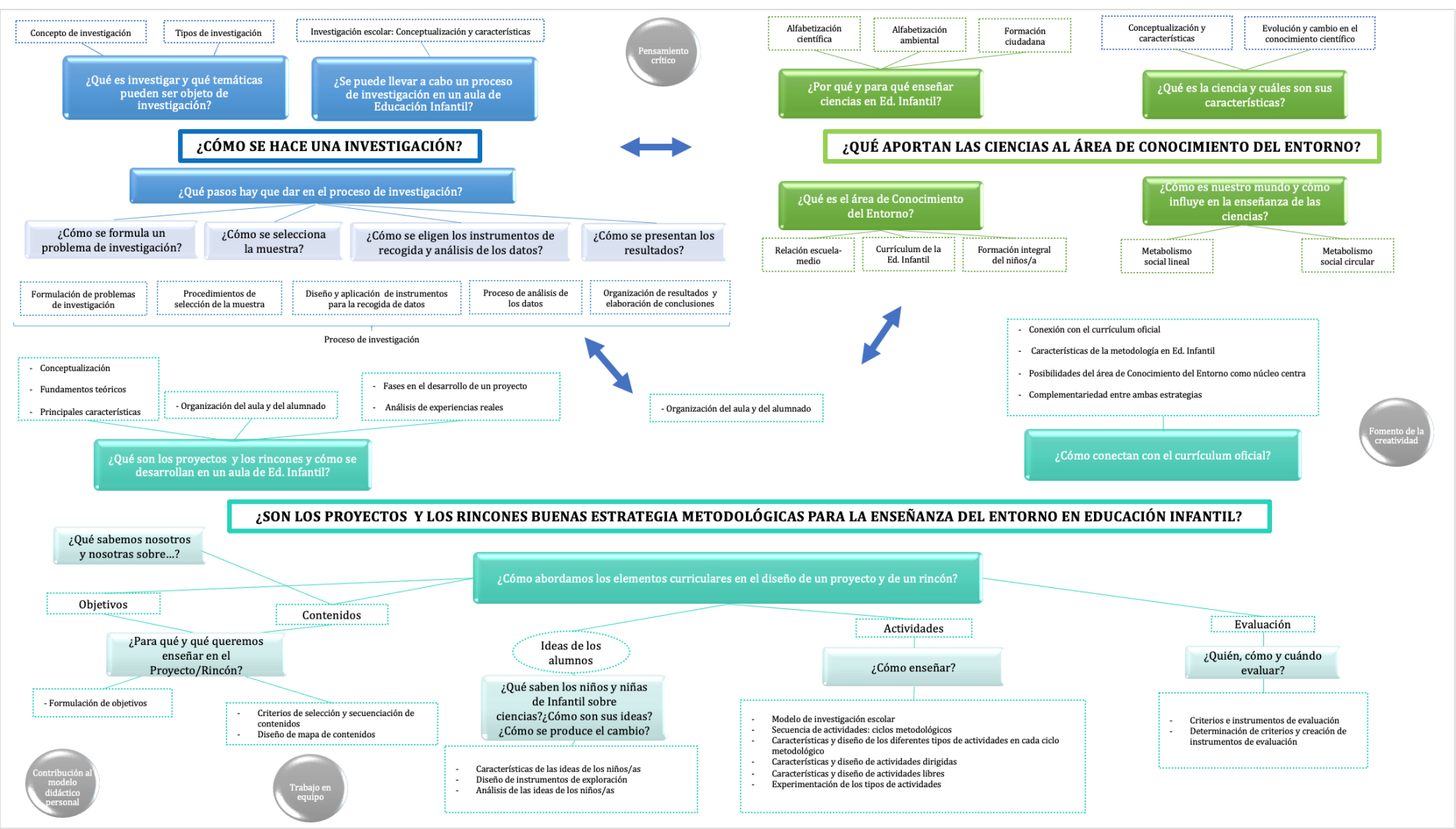

Figura 1. Mapa de contenidos

Ciclos de Mejora en el Aula (2020). Experiencias de Innovación Docente de la US (c) (i) $\ominus$ Esta obra se distribuye con la licencia Creative Commons 
El modelo metodológico que hasta ahora ha guiado nuestros CIMAS se ha centrado en establecer una estrecha relación entre teoría y práctica, siempre tomando como protagonista al alumnado. Para lograr esto, se trabaja en torno a problemas, que hay que identificar, para posteriormente secuenciar y elaborar una trama que nos sirva como guía en todo el proceso de aprendizaje. En lo que respecta al diseño de actividades, determinamos algunas características que deben cumplir: estar vinculadas con la práctica profesional, no tener una solución única y correcta, precisar de la reflexión y la construcción conjunta del conocimiento, considerar las ideas iniciales del alumnado, así como la evolución de las mismas y dar respuesta a las problemáticas planteadas. En este modelo, las clases son consideradas lugares y momentos en los que compartir ideas y construir entre todos nuevas formas de hacer frente a la realidad profesional. Para ello creemos esencial la creación de un buen clima de aula, donde el diálogo y la reflexión sean las herramientas principales. Así, en este modelo apostamos por comenzar acercándonos a las ideas del alumnado, introducir una actividad motivadora que capte su atención para iniciar el trabajo, ofrecer información nueva y favorecer el contraste entre esta y las ideas iniciales, para finalmente realizar tareas de recapitulación. En lo que respecta a la evaluación, los informes de trabajo son los principales instrumentos empleados, ya que permiten dejar plasmadas las diferentes actividades, las reflexiones generadas y el aprendizaje logrado.

Ciclos de Mejora en el Aula (2020). Experiencias de Innovación Docente de la US Esta obra se distribuye con la licencia Creative Commons 

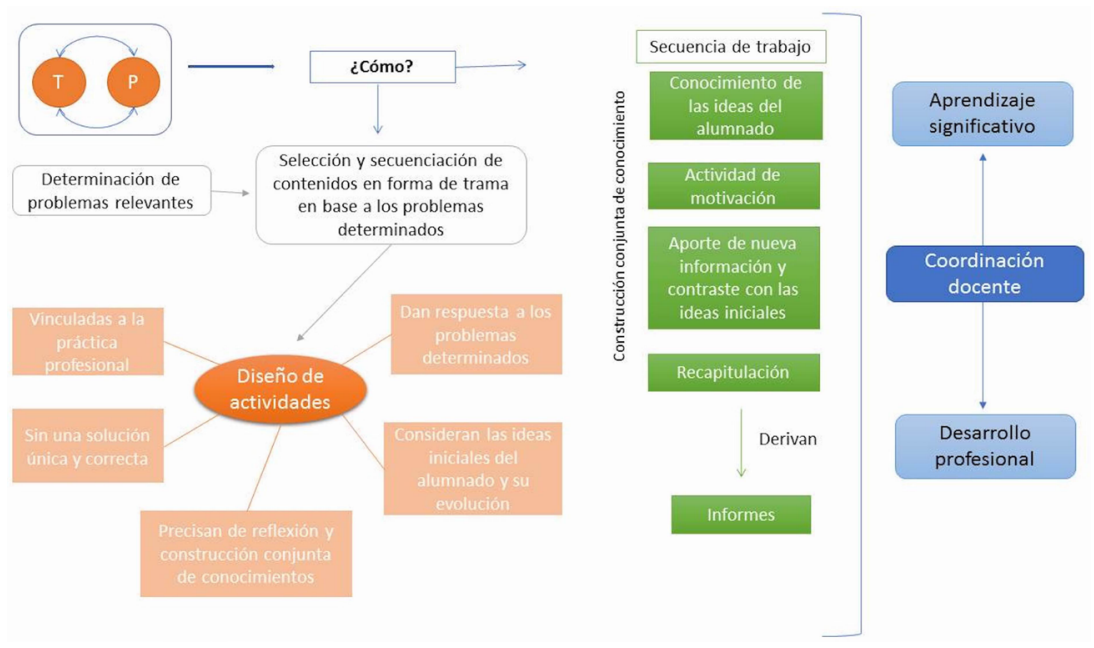

Figura 2. Modelo metodológico

Mostramos a continuación en forma de tablas, la secuencia de actividades, indicando el problema y/o subproblema con el que se relaciona, así como la fase del modelo metodológico al que pertencen (IA: Ideas de los alumnos, M: Motivación, C: Contraste, R: Recapitulación, IAF: Ideas de los alumnos Finales).

Ciclos de Mejora en el Aula (2020). Experiencias de Innovación Docente de la US (C) $($ ) $\Theta$ Esta obra se distribuye con la licencia Creative Commons 
Tabla 1. Secuencia de actividades Problema 1 de ambas materias

\begin{tabular}{|l|l|}
\hline $\begin{array}{l}\text { Problema: ¿Qué aportan las } \\
\text { ciencias al área de conocimiento } \\
\text { del Entorno? } \\
\text { Subproblema 1: ¿Qué es la ciencia y } \\
\text { cuáles son sus características? }\end{array}$ & $\begin{array}{l}\text { Problema: ¿Cómo se hace una } \\
\text { investigación? } \\
\text { Subproblema 1 y 2: ¿Qué es } \\
\text { investigar y qué temáticas pueden } \\
\text { ser objeto de investigación? ¿Qué } \\
\text { pasos hay que dar en el proceso de } \\
\text { investigación? }\end{array}$ \\
\hline FASE & \multicolumn{1}{|c|}{ ACTIVIDADES } \\
\hline IA
\end{tabular}

Ciclos de Mejora en el Aula (2020). Experiencias de Innovación Docente de la US Esta obra se distribuye con la licencia Creative Commons 


\begin{tabular}{|c|c|c|}
\hline$C$ & $\begin{array}{l}\text { SESIÓN } 3 \text { (Natural) } \\
\text { Semelweis ¿Cómo se } \\
\text { construye el conocimiento } \\
\text { científico? Actividad basada } \\
\text { en: Acebedo, García- } \\
\text { Carmona y Aragón (2016). } \\
\text { Puesta en común, } \\
\text { reelaboración esquema } \\
\text { construcción conocimiento } \\
\text { científico. } \\
\text { SESIóN 5 (Natural) } \\
\text { Actividad Experimental: } \\
\text { Hielo, basada en la } \\
\text { actividad ¿Lana o metal? De } \\
\text { Romero, Aguirre, Quesada y } \\
\text { Abril (2016). } \\
\text { Puesta en común y } \\
\text { reelaboración esquema } \\
\text { sobre la construcción del } \\
\text { conocimiento científico. } \\
\text { SESIóN 7 (Natural) } \\
\text { Reparto de textos para } \\
\text { debatir sobre ¿Es la ciencia } \\
\text { objetiva, verdadera y } \\
\text { neutra? } \\
\text { La maleta de Asimof } \\
\text { sobre cómo cambia el } \\
\text { conocimiento científico: } \\
\text { http://blogs.20minutos. } \\
\text { es/el-nutricionista-de- } \\
\text { la-general/2012/11/16/ } \\
\text { la-maleta-de-asimov-o- } \\
\text { por-que-lo-que-ayer-era- } \\
\text { bueno-hoy-es-malo-y- } \\
\text { viceversa/ }\end{array}$ & $\begin{array}{l}\text { SESIón } 4 \text { (Social) } \\
\text { - Selección de la temática por parte } \\
\text { de los grupos de trabajo. Cada grupo } \\
\text { elegirá una temática socio ambiental } \\
\text { de interés propio. } \\
\text { - Punto } 1 \text { del guion de trabajo: } \\
\text { Definición del problema de } \\
\text { investigación y justificación del } \\
\text { interés que tiene para el grupo. Se } \\
\text { trabaja con cada grupo particular } \\
\text { la manera de formular el problema, } \\
\text { haciendo una pequeña puesta en } \\
\text { común sobre ello una vez que todos } \\
\text { tienen delimitado lo que van a } \\
\text { trabajar. } \\
\text { - Inicio del punto } 2 \text { del guion } \\
\text { de trabajo: Planificación de la } \\
\text { investigación (fases del trabajo, } \\
\text { responsables de desarrollarlas, } \\
\text { fuentes de información que se van a } \\
\text { consultar, forma en la que se van a } \\
\text { recoger los datos....). } \\
\text { SESIón } 6 \text { (Social) } \\
\text { Desarrollo de la investigación por } \\
\text { parte de los grupos con seguimiento } \\
\text { por la profesora, atendiendo a los } \\
\text { puntos } 3,4 \text { y } 5 \text { del guion: } \\
\text { - Descripción de cada paso o } \\
\text { actividad realizada en el proceso } \\
\text { investigación. } \\
\text { - Conclusiones obtenidas, } \\
\text { tanto respecto a los resultados } \\
\text { propiamente dichos de la } \\
\text { investigación como respecto } \\
\text { al proceso de trabajo seguido. } \\
\text { Las conclusiones relativas a los } \\
\text { resultados deben presentarse } \\
\text { sintéticamente para cada problema. } \\
\text { - Reflexión sobre el propio proceso } \\
\text { de aprendizaje }\end{array}$ \\
\hline$R$ & $\begin{array}{l}\text { Puesta en común sobre las } \\
4 \text { preguntas iniciales sobre } \\
\text { la ciencia. }\end{array}$ & \\
\hline \multicolumn{2}{|c|}{$\begin{array}{l}\text { Subproblema 2: ¿Por qué y para } \\
\text { qué enseñar ciencias en Educación } \\
\text { Infantil? }\end{array}$} & \\
\hline
\end{tabular}

Ciclos de Mejora en el Aula (2020). Experiencias de Innovación Docente de la US Esta obra se distribuye con la licencia Creative Commons Reconocimiento-NoComercial-SinObraDerivada Internacional (CC BY-NC-ND 4.0.) 


\begin{tabular}{|c|c|c|}
\hline FASE & ACTIVIDADES & \\
\hline IA & $\begin{array}{l}\text { SESIÓN } 9 \text { (Natural) } \\
\text { Kahoo para conocer } \\
\text { las ideas iniciales del } \\
\text { alumnado respecto a este } \\
\text { subproblema }\end{array}$ & \\
\hline C & $\begin{array}{l}\text { Actividad en pequeños } \\
\text { grupos sobre razones por } \\
\text { las que las ciencias en } \\
\text { El son importantísimas } \\
\text { (Fragmentos de textos de } \\
\text { Eshach y Fried, } 2005 \text { y Khun, } \\
\text { 2012). }\end{array}$ & $\begin{array}{l}\text { SESIÓN } 8 \text { (Social) } \\
\text { Desarrollo de la investigación por } \\
\text { parte de los grupos con seguimiento } \\
\text { por la profesora. }\end{array}$ \\
\hline $\mathrm{R} / \mathrm{IAF}$ & $\begin{array}{l}\text { Puesta en común } \\
\text { y realización de } \\
\text { conclusiones. }\end{array}$ & \\
\hline \multicolumn{2}{|c|}{$\begin{array}{l}\text { Subproblema 3: ¿Cómo es nuestro } \\
\text { mundo y cómo influye en la } \\
\text { Enseñanza de las Ciencias? }\end{array}$} & \\
\hline IA & $\begin{array}{l}\text { SESIÓN } \mathbf{1 0} \text { (Natural) } \\
\text { Presentación de las ideas } \\
\text { iniciales respecto a este } \\
\text { subproblema y puesta en } \\
\text { común. } \\
\text { Síntesis de las } \\
\text { Investigaciones } \\
\text { sobre problemas } \\
\text { socioambientales que } \\
\text { están realizando en la } \\
\text { asignatura de Conocimiento } \\
\text { del Entorno Social. ¿Qué } \\
\text { datos nos faltan aún, que } \\
\text { no he comprendido, qué } \\
\text { dudas tengo? }\end{array}$ & \\
\hline
\end{tabular}

Ciclos de Mejora en el Aula (2020). Experiencias de Innovación Docente de la US Esta obra se distribuye con la licencia Creative Commons 


\begin{tabular}{|c|c|c|}
\hline \multirow[t]{2}{*}{ C } & \multirow[t]{2}{*}{$\begin{array}{l}\text { Actividad de la silla } \\
\text { (Ideas, sf) y/o Calculamos } \\
\text { nuestra Huella de Carbono } \\
\text { (Observatorio CO2 de la } \\
\text { cátedra de ética ambiental } \\
\text { UAH-FTPGB). }\end{array}$} & $\begin{array}{l}\text { SESIÓN } 11 \text { (Social) } \\
\text { Exposición de las investigaciones } \\
\text { mediante vídeos breves que } \\
\text { quedarán subidos a la plataforma } \\
\text { y deberán valorar ofreciendo un } \\
\text { breve comentario crítico acerca de } \\
\text { la investigación realizada por los } \\
\text { compañeros. }\end{array}$ \\
\hline & & $\begin{array}{l}\text { Subproblema 3: ¿Se puede llevar a } \\
\text { cabo un proceso de investigación en } \\
\text { un aula de Educación Infantil? }\end{array}$ \\
\hline Cy R & $\begin{array}{l}\text { SESIÓN } 12 \text { (Natural) } \\
\text { Rescatando las temáticas } \\
\text { principales de las } \\
\text { investigaciones realizadas } \\
\text { en las investigaciones de } \\
\text { los alumnos, se realiza } \\
\text { una exposición dialogada } \\
\text { sobre el diagnóstico de la } \\
\text { Situación del mundo. }\end{array}$ & $\begin{array}{l}\text { Sesión } 13 \text { (Social) } \\
\text { Conexión de la experiencia de } \\
\text { investigación con el trabajo en } \\
\text { Educación Infantil. Debate en } \\
\text { torno a la lectura del documento: } \\
\text { Tonucci, F. (2001) ¿Cómo introducir la } \\
\text { investigación escolar? Investigación } \\
\text { en la Escuela, 43, 39-50. } \\
\text { Todos los grupos recogerán una } \\
\text { breve reflexión sobre este tema en su } \\
\text { trabajo de investigación. }\end{array}$ \\
\hline \multicolumn{3}{|c|}{ Subproblema 4: ¿Qué es el área de conocimiento del Entorno? } \\
\hline FASE & \multicolumn{2}{|c|}{ ACTIVIDADES } \\
\hline IA & \multicolumn{2}{|c|}{$\begin{array}{l}\text { SESIÓN } \mathbf{1 4} \text { (Natural) } \\
\text { Debate para conocer las ideas iniciales respecto a este problema y } \\
\text { puesta en común. }\end{array}$} \\
\hline C & \multicolumn{2}{|c|}{$\begin{array}{l}\text { Análisis del curriculum siguiendo guion de trabajo. ¿Qué nos dice } \\
\text { la legislación sobre el Para qué y el qué enseñar en el área de } \\
\text { Entorno? ¿Qué estrategias metodológicas propone? } \\
\text { Partiendo de la Orden de } 5 \text { de agosto, por la que se desarrolla el } \\
\text { Currículo correspondiente a la Educación Infantil en Andalucía: } \\
\text { 1. Identificar y listar los objetivos de etapa y objetivos de área. } \\
\text { 2. Los contenidos a abordar en esta área provienen de diferentes } \\
\text { disciplinas ¿De cuáles? ¿Ha sido fácil identificarlas? } \\
\text { 3. Listar los contenidos mínimos a tratar en el Área de Conocimiento } \\
\text { del Entorno, utilizando palabras claves. } \\
\text { 4. Identificar las estrategias metodológicas propuestas por la } \\
\text { normativa. } \\
\text { SESIón } \mathbf{1 5} \text { (Social) y } \mathbf{1 6} \text { (Natural) } \\
\text { Continuamos con la actividad de análisis del currículum. }\end{array}$} \\
\hline
\end{tabular}

Ciclos de Mejora en el Aula (2020). Experiencias de Innovación Docente de la US Esta obra se distribuye con la licencia Creative Commons Reconocimiento-NoComercial-SinObraDerivada Internacional (CC BY-NC-ND 4.0.) 


\begin{tabular}{|l|l|}
\hline$R$ & $\begin{array}{l}\text { SESIÓN 17 (Social) } \\
\text { Cerramos la actividad de análisis, y realizamos la puesta en } \\
\text { común. Recomendación para casa de la lectura Capítulo 1 de Puig y } \\
\text { Rodríguez-Marín, 2018. }\end{array}$ \\
\hline IAF & Realización cuestionario Final Bloque 1. \\
\hline
\end{tabular}

Tabla 2. Secuencia de actividades Problema 2 de ambas materias

Problema General: ¿Son los proyectos y los rincones buenas estrategias
metodológicas para la enseñanza del Entorno en Educación Infantil?

\begin{tabular}{|c|c|}
\hline FASE & ACTIVIDADES \\
\hline IA & $\begin{array}{l}\text { SESIÓN } 1 \text { (Natural) } \\
\text { Cuestionario Inicial sobre los proyectos y los rincones. } \\
\text { Puesta en común preguntas cuestionario relacionadas con el para } \\
\text { qué y el qué enseñar. } \\
\text { Exposición dialogada sobre los proyectos basado en las lecturas } \\
\text { de: Pozuelo (2007), Benítez (2008), Feito (2006), Vizcaino (2008) y } \\
\text { Muñoz y Díaz (2009). }\end{array}$ \\
\hline $\mathrm{IA}$ & $\begin{array}{l}\text { SESIÓN } 2 \text { (Social) } \\
\text { Exposición dialogada sobre los rincones de trabajo basada en las } \\
\text { lecturas de: Madrid y Mayorga (2012) y Laguía y Vidal, (2013 ). }\end{array}$ \\
\hline \multicolumn{2}{|c|}{ Problema 0: ¿Qué sabemos nosotros y nosotras sobre....? } \\
\hline FASE & ACTIVIDADES \\
\hline C & $\begin{array}{l}\text { SESIÓN } 3 \text { (Natural) } \\
\text { La primera tarea a realizar por los alumnos/as es la elección de } \\
\text { una temática basándose en el listado realizado al finalizar el } \\
\text { bloque anterior y/o de un listado que ofrecen las docentes. } \\
\text { A partir de esa elección se procede a la elaboración de un } \\
\text { dossier sobre la temática, para ello se ofrece guion de trabajo en } \\
\text { el que deben plantear un índice de su temática y un esquema } \\
\text { final. } \\
\text { SESIONES } \mathbf{4} \text { y } \mathbf{5} \text { (Social y Natural) } \\
\text { Trabajo en clase sobre el dossier. }\end{array}$ \\
\hline
\end{tabular}

Ciclos de Mejora en el Aula (2020). Experiencias de Innovación Docente de la US Esta obra se distribuye con la licencia Creative Commons Reconocimiento-NoComercial-SinObraDerivada $\quad 4.0$ Internacional (CC BY-NC-ND 4.0.) 


\begin{tabular}{|l|l|}
\hline R / IAF & SESIÓN 6 (Natural) \\
& Trabajo en clase sobre el dossier. Subida a la plataforma de los \\
& esquédéditos
\end{tabular}
esquemas finales de cada temática para que puedan compartirlo con los compañeros.

\section{SESIÓN 7 (Social)}

Puesta en común sobre aspectos que le han llamado la atención y dudas que han quedado.

\section{Problema 1: ¿Para qué y qué queremos enseñar en el Proyecto/Rincón?}

\begin{tabular}{l|l} 
IA & SESIÓN 8 (Social)
\end{tabular}

Redactamos objetivos y seleccionamos contenidos teniendo en cuenta diferentes criterios: ciencia, contexto, legislación, Realizamos un listado. Seleccionamos aquellos más vinculados al rincón y los más vinculados al proyecto.

\section{SESIÓN 9 (Natural) y SESION 10 (Social)}

Exposición dialogada sobre la presentación de los contenidos: Mapas de problemas-contenidos y ejemplos concretos desde cada una de las disciplinas.

\section{SESIÓN 11 (Natural)}

Continuamos con la redacción de objetivos y contenidos. Diseño de mapas-problemas.

\section{SESIÓN 12 (Social)}

Diseño de mapas-problemas.

\section{R/ IF SESIÓN 13 (Natural)}

Puesta en común mapas. Los alumnos deberán tener subido a la plataforma sus mapas de forma que, en esta puesta en común, la docente realizará una selección de algunos de los mapas y los grupos que los hayan elaborado podrán ir comentándolos. Cuestionario final bloque II.

Problema 2. Ideas de los alumnos. Subproblema 1 y 2: ¿Qué saben los niños y niñas de Infantil sobre ciencias? ¿Cómo son sus ideas?

¿Cómo podemos averiguarlo? ¿Para qué nos sirve?

\begin{tabular}{|l|l|}
\hline FASE & \multicolumn{1}{|c|}{ ACTIVIDADES } \\
\hline IA & $\begin{array}{l}\text { SESIÓN 1(Social) } \\
\text { Puesta en común preguntas del cuestionario inicial sobre } \\
\text { proyectos y rincones relacionados con el bloque III: las ideas de } \\
\text { los alumnos. }\end{array}$
\end{tabular}

Ciclos de Mejora en el Aula (2020). Experiencias de Innovación Docente de la US Esta obra se distribuye con la licencia Creative Commons Reconocimiento-NoComercial-SinObraDerivada 4.0 Internacional (CC BY-NC-ND 4.0.) 


\begin{tabular}{|c|c|}
\hline$M$ & $\begin{array}{l}\text { Video "Construyendo nuestro mundo" (Cubero, 2006) y “Ideas de } \\
\text { los alumnos sobre cuestiones sociales" (Puig, 2018). Visualización } \\
\text { con breves comentarios. } \\
\text { Proponemos la actividad que va a organizar este bloque: } \\
\text { Investigar (diseñar y analizar) las ideas de los alumnos/as sobre } \\
\text { los problemas seleccionados. Presentación del informe de } \\
\text { este bloque. En el marco de la asignatura de Conocimiento del } \\
\text { Entorno Social se trabajarán las características del pensamiento } \\
\text { infantil y en el marco de la asignatura de Enseñanza del Entorno } \\
\text { Natural la categorización de las respuestas basadas en Hipótesis } \\
\text { de Transición o Progresión (Rodríguez-Marín, Fernández-Arroyo y } \\
\text { García, 2014). }\end{array}$ \\
\hline & $\begin{array}{l}\text { SESIÓN } \mathbf{2} \text { (Natural) } \\
\text { Exposición dialogada sobre cómo podemos averiguar las ideas de } \\
\text { los alumnos. Indicaciones sobre tipos de instrumentos y formas } \\
\text { de realizarlo. Planteamos ejemplos concretos de instrumentos (1 } \\
\text { h y } 20 \text { min) } \\
\text { El alumnado comenzará eligiendo la muestra (4-5 Niños/as de } \\
\text { la misma edad entre } 3 \text { y } 6 \text { años) y definiendo el instrumento } \\
\text { a elegir. Comienzo del diseño del instrumento de recogida de } \\
\text { datos. } \\
\text { SESIÓN } \mathbf{3} \text { (Social) } \\
\text { Diseño instrumento de recogida de datos. } \\
\text { SESIÓN } \mathbf{5} \text { (Natural) } \\
\text { Exposición dialogada sobre algunas claves más sobre cómo } \\
\text { realizar el instrumento, teniendo en cuenta las características de } \\
\text { las ideas de los niños en general. } \\
\text { Continuar con el diseño del instrumento. } \\
\text { SESIÓN } \mathbf{6} \text { (Social) } \\
\text { Continuar con el diseño instrumento. } \\
\text { Puesta en común de algunos de los instrumentos para reformular } \\
\text { preguntas si fuese necesario. } \\
\text { SESIóN } 7 \text { (Natural) y SESIóN } \mathbf{8} \text { (Social) } \\
\text { Recogida de datos. } \\
\text { SESIÓN } \mathbf{9} \text { (Natural) } \\
\text { Transcripción de las entrevistas y/o asambleas. } \\
\text { SESIÓN } \mathbf{1 0} \text { (Social) } \\
\text { Exposición dialogada sobre como analizar las respuestas de los } \\
\text { niños/as en torno a las características del pensamiento infantil y } \\
\text { su utilización didáctica. } \\
\text { SESIóN } \mathbf{1 1 ~ ( N a t u r a l ) ~} \\
\text { Exposición dialogada sobre como analizar las respuestas de los } \\
\text { niños/as estableciendo categorías e hipótesis de progresión. } \\
\text { Comienzo de ejemplo práctico del análisis por categorías y } \\
\text { comparación con otras investigaciones. }\end{array}$ \\
\hline
\end{tabular}

Ciclos de Mejora en el Aula (2020). Experiencias de Innovación Docente de la US Esta obra se distribuye con la licencia Creative Commons Reconocimiento-NoComercial-SinObraDerivada $\quad 4.0$ Internacional (CC BY-NC-ND 4.0.) 


\begin{tabular}{|c|c|}
\hline C & $\begin{array}{l}\text { SESIÓN } 12 \text { (Social) } \\
\text { Análisis de las respuestas de los niños/as (pensamiento infantil). } \\
\text { SESIÓN } \mathbf{1 3} \text { (Natural) } \\
\text { Análisis de las respuestas de los niños/as (categorías). } \\
\text { SESIÓN } \mathbf{1 4} \text { (Social) y SESIÓN } \mathbf{1 5} \text { (Natural) } \\
\text { Continuación de los análisis de las respuestas de los niños/as } \\
\text { (categorías y pensamiento infantil). }\end{array}$ \\
\hline $\mathrm{R}$ & $\begin{array}{l}\text { SESIÓN } 16 \text { (Social) } \\
\text { Puesta en común de algunos de los análisis realizados. } \\
\text { SESIÓN } 17 \text { (Natural) } \\
\text { Puesta en común de algunos de los análisis realizados. }\end{array}$ \\
\hline \multicolumn{2}{|c|}{ Subproblema 3: ¿Cómo se produce el cambio en las Ideas de los niños/as? } \\
\hline FASE & ACTIVIDADES \\
\hline M & $\begin{array}{l}\text { SESIÓN } 18 \text { (Social) } \\
\text { Kahoo con una selección de preguntas del cuestionario inicial. } \\
\text { Ponemos los resultados anteriores y comparamos con los } \\
\text { actuales. }\end{array}$ \\
\hline IA & $\begin{array}{l}\text { Les preguntamos ¿Si han cambiado sus ideas y por qué creen que } \\
\text { han cambiado? Diálogo y debate. } \\
\text { El tiempo restante se dejará para resolver dudas respecto a la } \\
\text { actividad de análisis de las ideas de los niños. }\end{array}$ \\
\hline C & $\begin{array}{l}\text { SESIÓN } 19 \text { (Natural) } \\
\text { Les pasamos unos esquemas sobre lo que ocurre con la } \\
\text { información cuando llega a nuestro cerebro ( } 3 \text { casos) Trabajo en } \\
\text { grupo. } \\
\text { Puesta en común y exposición dialogada sobre el cambio en las } \\
\text { Ideas de los niños/as. }\end{array}$ \\
\hline $\mathrm{R} / \mathrm{IAF}$ & $\begin{array}{l}\text { Realización del cuestionario final individual del bloque } 3 \text { sobre } \\
\text { las ideas de los alumnos. }\end{array}$ \\
\hline \multicolumn{2}{|c|}{ Problema 3: ¿Cómo enseñar? } \\
\hline FASE & ACTIVIDADES \\
\hline IA & $\begin{array}{l}\text { SESIÓN } \mathbf{1} \text { (Social) } \\
\text { Exposición dialogada sobre la globalización en infantil y los } \\
\text { rincones } \\
\text { Lectura de rincones, y tipos de actividades Capítulos 7, 8, } 9 \text { y } 10 \\
\text { del libro Puig, M. y Rodríguez-Marín, F. (Coords.) La enseñanza } \\
\text { del entorno en Educación Infantil. Proyectos y rincones. Madrid: } \\
\text { Pirámide. } \\
\text { SESIÓN } \mathbf{2} \text { (Natural) } \\
\text { Puesta en común preguntas del cuestionario inicial sobre } \\
\text { proyectos relacionados con el bloque IV: cómo enseñar y } \\
\text { presentación informe final. }\end{array}$ \\
\hline
\end{tabular}

Ciclos de Mejora en el Aula (2020). Experiencias de Innovación Docente de la US Esta obra se distribuye con la licencia Creative Commons Reconocimiento-NoComercial-SinObraDerivada Internacional (CC BY-NC-ND 4.0.) 


\begin{tabular}{|c|c|}
\hline C & $\begin{array}{l}\text { Lectura de proyectos: Fragmento del capítulo } 1 \text { del libro Puig, } \\
\text { M. y Rodríguez-Marín, F. (Coords.) La enseñanza del entorno en } \\
\text { Educación Infantil. Proyectos y rincones. Madrid: Pirámide. } \\
\text { Capítulos 2, 3, 4, } 5 \text { y } 6 \text { del libro Puig, M. y Rodríguez-Marín, } \\
\text { F. (Coords.) La enseñanza del entorno en Educación Infantil. } \\
\text { Proyectos y rincones. Madrid: Pirámide } \\
\text { Exposición dialogada metodología Investigativa/ Secuencia (1h). } \\
\text { SESIóN } \mathbf{3} \text { (Social) } \\
\text { Exposición dialogada Rincones } \\
\text { SESıóN } \mathbf{4} \text { (Natural) } \\
\text { Taller Actividades: presentación de una selección de actividades } \\
\text { y recursos. } \\
\text { SESIóN } \mathbf{5} \text { (Social) } \\
\text { Taller Actividades: presentación de una selección de actividades } \\
\text { libres y dirigidas y recursos. } \\
\text { SESIóN } \mathbf{6} \text { (Natural) } \\
\text { Presentación de varios videos de docentes que muestran cómo } \\
\text { trabajan proyectos y rincones. } \\
\text { Presentación del trabajo: Diseño de una secuencia sobre uno de } \\
\text { los problemas del mapa inicial. } \\
\text { SESıóN } 7 \text { (Social) } \\
\text { Diseño actividades del rincón. } \\
\text { SESIóN } \mathbf{8} \text { (Natural) } \\
\text { Diseño actividades del proyecto. }\end{array}$ \\
\hline $\mathrm{R}$ & $\begin{array}{l}\text { SESIÓN } \mathbf{9} \text { (Social) y } \mathbf{1 0} \text { (Natural) SESIÓN } \mathbf{1 1} \text { (Social) y SESIÓN } 12 \\
\text { (Natural) } \\
\text { Diseño de actividades del proyecto y del rincón. Creación de un } \\
\text { blog en el que recoger todo lo diseñado. }\end{array}$ \\
\hline $\mathrm{R} / \mathrm{IAF}$ & $\begin{array}{l}\text { SESIÓN } 13 \text { (Social) } \\
\text { Valoración de los blogs de los compañeros. } \\
\text { Resolución de dudas actividades rincón. } \\
\text { SESIÓN } \mathbf{1 4} \text { (Natural) } \\
\text { Resolución de dudas secuencia de actividades. } \\
\text { Realización Cuestionario final relacionado con los contenidos del } \\
\text { bloque IV. }\end{array}$ \\
\hline \multicolumn{2}{|c|}{ Problema 4: ¿Quién, cómo y cuándo evaluar? } \\
\hline FASE & ACTIVIDADES \\
\hline IA & $\begin{array}{l}\text { SESIÓN } \mathbf{1} \text { (Social) } \\
\text { Puesta en común preguntas del Cuestionario Inicial relacionado } \\
\text { con la evaluación }\end{array}$ \\
\hline
\end{tabular}

Ciclos de Mejora en el Aula (2020). Experiencias de Innovación Docente de la US Esta obra se distribuye con la licencia Creative Commons Reconocimiento-NoComercial-SinObraDerivada $\quad 4.0$ Internacional (CC BY-NC-ND 4.0.) 


\begin{tabular}{|c|c|}
\hline$C$ & $\begin{array}{l}\text { Revisamos las lecturas realizadas en el bloque anterior y } \\
\text { prestamos atención a la forma en la que se aborda en las } \\
\text { experiencias descritas la evaluación. } \\
\text { SESIóN } \mathbf{2} \text { (Natural) } \\
\text { Puesta en común y extracción de conclusiones. } \\
\text { Lectura del documento: } \\
\text { Pellicer, C. (2017). La evaluación del aprendizaje en Educación } \\
\text { Infantil (Capítulos 1, } 3 \text { y 4). Madrid: Anaya. } \\
\text { SESIóN } \mathbf{3} \text { (Social) } \\
\text { Exposición dialogada sobre el sistema de evaluación: criterios, } \\
\text { momentos, instrumentos y sistema de calificación, así como } \\
\text { sobre las funciones de la evaluación. } \\
\text { SESIón } \mathbf{5} \text { (Social) } \\
\text { Elaboración de criterios de evaluación para el rincón y proyecto } \\
\text { diseñado. } \\
\text { SESIóN } \mathbf{6} \text { (Natural) } \\
\text { Elaboración de criterios de evaluación para el rincón y proyecto } \\
\text { diseñado. }\end{array}$ \\
\hline $\mathrm{R}$ & $\begin{array}{l}\text { SESIÓN } 8 \text { (Social) } \\
\text { Puesta en común de algunos grupos sobre el sistema de } \\
\text { evaluación. }\end{array}$ \\
\hline
\end{tabular}

Tabla 3. Secuencia de actividades finales

\section{ACTIVIDADES FINALES}

\section{SESIÓN 1 (Natural)}

Consulta de dudas y tiempo de trabajo para el cierre del Informe Final (Proyecto).

\section{SESIÓN 2 (Social)}

Consulta de dudas y tiempo de trabajo para el cierre del Informe Final (Rincón).

\section{SESIÓN 3, 4, 5 y 6 (Natural y Social)}

Preparación exposición sobre los rincones en formato vídeo.

\section{SESIÓN 7 y 8 (Natural y Social)}

Subida de vídeos a la plataforma para visionado y valoración de todos.

Determinación de las bases para la valoración y votación de los mejores rincones.

Cuestionario Final sobre los proyectos y rincones.

\section{SESIÓN 9 (Natural/Social)}

Reparto de premios Rincones. Evaluación de las asignaturas.

Ciclos de Mejora en el Aula (2020). Experiencias de Innovación Docente de la US Esta obra se distribuye con la licencia Creative Commons 
Cuestionario inicial-final para hacer un seguimiento de la evolución de los modelos mentales de los estudiantes

Presentamos a continuación el instrumento que emplearemos para recoger las ideas iniciales y finales para cada uno de los Bloques/problemas:

Bloque I: ¿Qué aportan las ciencias al área de Conocimiento del Entorno en Infantil? (E. Natural) y ¿Cómo se hace una investigación? (E. Social)

1. Me gustaría que definieses con tus palabras iqué es ciencia?

Objetivo: Grado de reconocimiento del concepto de ciencia. Problema 1, subproblema 1 (E. Natural).

2. ¿Cómo se construye el conocimiento científico? ¿Cuáles son los pasos de una investigación científica?

Objetivo: Grado de conocimiento de las fases de construcción del conocimiento científico. Problema 1, subproblema 1 (E. Natural) y subproblema 1 y 2 (E. Social).

3. Indica 3 problemas socioambientales más relevantes en el momento actual, defínelos con tus palabras y justifica por qué lo has elegido

Objetivo: Grado de conocimiento de los problemas del mundo. Problema 1, subproblema 3 (E. Natural).

4. La ciencia y la situación actual de nuestro mundo afecta a la educación que tenemos que dar a nuestros niños y niñas ¿En qué? Pon algún ejemplo concreto Objetivo: Relación ciencia, situación del mundo y educación. Problema 1, subproblema 3 E. Natural) y subproblema 3 (E. Social).

Bloque 2: ¿Son los proyectos y los rincones buenas estrategias metodológicas para la enseñanza del Entorno en Educacón Infantil?

Ciclos de Mejora en el Aula (2020). Experiencias de Innovación Docente de la US Esta obra se distribuye con la licencia Creative Commons 
Dentro de poco comenzarás las prácticas, imagínate que en el cole al que vas a ir te comentan que están trabajando por proyectos:

1. ¿Sabrías comentarnos en qué consiste trabajar por proyectos?

Objetivo: Grado de conocimiento sobre la estrategia metodológica de los proyectos. Problema General. Bloque 1.

2. ¿Qué pasos tendrías que seguir para llevarlo a cabo? Objetivo: Grado de conocimiento sobre la estrategia metodológica de los proyectos. Problema General. Bloque 1.

3. Y si además de indican que organizan el aula por rincones, ¿sabrías a qué se refieren? Explícalo.

Objetivo: Grado de conocimiento sobre la estrategia metodológica de los rincones. Problema General. Bloque 1.

4. Estarás de acuerdo en que, tanto a través de los proyectos como de los rincones, se trabajan diversos contenidos, pero ¿de qué tipo son los contenidos que se pretenden enseñar?
a) Son casi todos conceptos
b) Son sobre todo procedimentales y actitudes
c) Son conceptos y también procedimientos y actitudes
d) Otros

Objetivo: Reconocimiento de los tipos de contenidos. Problema General. Bloque 1.

5. ¿Cómo se organizan y presentan a los alumnos los contenidos?
a) Como un conjunto de problemas abiertos
b) Son sobre todo procedimentales y actitudes
c) Son conceptos y también procedimientos y actitudes

Ciclos de Mejora en el Aula (2020). Experiencias de Innovación Docente de la US Esta obra se distribuye con la licencia Creative Commons 
d) Otros

Objetivo: Organización de los contenidos. Problema General. Bloque 1.

6. ¿Qué entiendes por ideas de los alumnos?

a) Son los conocimientos previos que los alumnos deben tener para aprender un tema

b) Las ideas de los alumnos son un conocimiento diferente al conocimiento científico y escolar, que utilizan en su vida cotidiana

c) En realidad los alumnos no tienen ideas sobre los contenidos de ciencias y si las tienen son erróneas y sin interés para la enseñanza y el aprendizaje

d) Otros

Objetivo: Grado de conocimiento de las ideas de los alumnos. Problema General. Bloque 2.

7. ¿Qué crees que ocurre con las ideas de los alumnos en la escuela?

a) Se supone que los alumnos cambian sus ideas por lo que queremos enseñarles

b) Se supone que los alumnos reelaboran sus propias ideas al interaccionar con nuevas informaciones

c) Se supone que los alumnos añaden lo que les hemos enseñado a sus propias ideas

d) Otros

Objetivo: Grado de conocimiento de las ideas de los alumnos. Problema General. Bloque 2.

8. ¿Cuándo y para qué se pueden tener en cuenta?

a) A lo largo de todas las clases, primero para detectarlas, luego para compararlas con otras informaciones y finalmente para reelaborarlas.

b) Al principio y al final de las clases para saber que es lo que han aprendido. 

c) Al principio, para saber lo que los alumnos sa- ben del tema y así insistir en lo que tienen más errores
d) Otros

Objetivo: Grado de conocimiento de las ideas de los alumnos. Problema General. Bloque 2.

9. ¿Qué tipo de actividades se podrían hacer en un proyecto? Pon dos ejemplos diferentes

Objetivo: Grado de conocimiento de la tipología de actividades. Problema General. Bloque 3.

10. Y en un rincón ¿qué tipo de actividades se podrían realizar? Pon dos ejemplos distintos.

Objetivo: Grado de conocimiento de la tipología de actividades. Problema General. Bloque 3.

11. De qué manera organizas las actividades que vas a llevar a cabo. ¿Qué criterio utilizas?

Objetivo: Grado de conocimiento del proceso metodológico. Problema General. Bloque 3.

12. Los proyectos y los rincones ¿se evalúan? ¿De qué manera? Desarrolla esta pregunta con tus palabras

Objetivo: Grado de conocimiento de la evaluación de los proyectos. Problema General. Bloque 4.

\section{Aplicación del CIMA}

El desarrollo del CIMA ha estado principalmente marcado por las dificultades encontradas a la hora de gestionar la situación de semipresencialidad en un primer momento y de virtualidad posteriormente. A pesar de partir de un diseño previo muy detallado, hemos encontrado dificultades para cuadrarlo en el tiempo con las circunstancias que nos han ido rodeando. De esta forma, aunque hemos conseguido realizarlo respetando la secuencia, hemos tenido sesiones que se han prologando y que en

Ciclos de Mejora en el Aula (2020). Experiencias de Innovación Docente de la US Esta obra se distribuye con la licencia Creative Commons 
lugar de ocupar una hora y veinte como teníamos planificado, han tenido que realizarse en dos horas cuarenta minutos, como ha sido el caso de las sesiones 1, 4 y 11. Con el fin de poder seguir la secuencia prevista, fuimos retrasando las siguientes sesiones sin modificar su orden y contenido. Esto, sumado a que la primera semana del curso tuvimos que dedicarla por completo a presentar las asignaturas y tener al menos un primer contacto con cada uno de los subgrupos en los que se dividía el grupo clase, nos han llevado a tener un retraso algo considerable respecto a la planificación inicial, lo que ha hecho que en las fechas en las que debíamos analizar los cuestionarios iniciales y finales del alumnado, solo dispusiéramos del bloque 1 completo.

En cuanto al desarrollo general del CIMA, consideramos que ha funcionado de forma adecuada, pero que el escenario de semipresencialidad y sobre todo el de virtualidad, han entorpecido mucho la participación de los estudiantes. La creación de los grupos en la plataforma, dotándoles de un espacio propio ha sido para nosotras una de los principales recursos para poder desarrollar las asignaturas. Con esta herramienta hemos podido hacer el seguimiento que veníamos realizando, asesorando a cada grupo en base al trabajo realizado, sin embargo, hemos percibido mayores dificultades para comprender el sentido de las actividades propuestas y seguir el hilo de las clases. Con frecuencia las preguntas realizadas hacían alusión a aspectos comentados previamente, pero que habían pasado desapercibidos. A fin de ir solventado estas dificultades hemos creado pequeñas guías a modo de infografías que permitieran recoger todo lo que debían hacer a lo largo de cada semana.

Respecto al contenido, en relación al problema ¿Cómo se hace una investigación? hemos observado dificultades para afrontar el proceso de investigación de manera 
autónoma y principalmente para definir el problema inicial que diera lugar a la investigación, de ahí que se tuviera que dedicar más tiempo del previsto a esto. Quizás pudiera ser interesante en posteriores cursos, presentar a modo de ejemplo algunas problemáticas. Además, otro obstáculo ha estado en la falta de reflexión y la necesidad que suelen manifestar por querer vincular cualquier actividad que realicen con su papel como maestros/as diseñadores de actividades. Aunque esto ha sido habitual en otros cursos, este año nos ha resultado especialmente significativo.

En relación con el problema, ¿Qué aportan las ciencias al área de conocimiento del Entorno?, se han ido realizando las actividades propuestas a excepción de la actividad experimental de la sesión 5 y el juego de simulación de la sesión 10. No se han sustituido ni adaptado por otra actividad, debido a los retrasos comentados anteriormente, y porque en la primera de las actividades necesitaba de materiales propios del laboratorio que no podíamos compartir en estos momentos, y la segunda, implicaba movimiento del alumnado por el aula, acciones que tampoco podíamos hacer.

En cuanto al subproblema ¿qué es el área de conocimiento del entorno?, creemos que el análisis solicitado ha resultado adecuado para profundizar en el currículum, puesto que no suelen trabajar con la normativa y se han mostrado en ocasiones sorprendidos al descubrir las múltiples posibilidades que ofrece.

\section{Evaluación del aprendizaje de los estudiantes}

Para determinar el aprendizaje de los alumnos/as, se diseñaron diferentes cuestionarios que se han recogido en un apartado anterior. Dadas las limitaciones de 
tiempo, solo ofrecemos aquí los resultados del cuestionario correspondiente al bloque 1 de ambas materias, que fue aplicado de forma individual al inicio y al final del bloque. Los datos obtenidos en ambos momentos nos permiten conocer la evolución de los aprendizajes. Una vez que los alumnos/as completaron el cuestionario, analizamos sus respuestas determinando niveles de complejidad, de forma que ordenamos las respuestas de las más simples a las más complejas y calculamos el porcentaje de alumnos/as que estaba en cada nivel. A continuación, podemos ver las escaleras de aprendizaje, en las que es posible identificar cada uno de los niveles, así como el porcentaje de alumnos/as que se sitúa en ellos al inicio y al final del bloque 1.

Observamos en la figura 3, que hay un avance entre el cuestionario inicial y final respecto al grado de conocimiento del concepto de ciencia. Al inicio de la intervención, encontramos respuestas que se posicionaban en los primeros escalones de la escalera, estando el 79,25\% de las respuestas en el nivel 1, haciendo una definición muy escueta de la ciencia, del tipo "Análisis de todas las cosas" (A.3). Al final de la intervención, encontramos respuestas en los tres niveles, pero más de la mitad de la clase se encuentran entre el nivel 2 y 3 . Un $21,52 \%$ de las respuestas se sitúan en el nivel más complejo, en sus definiciones, hacen alusión a referencias que han consultado o incluso a las aportaciones hechas en clase, teniendo respuestas del tipo:

Según lo aprendido en esta asignatura y contrastándolo con los apuntes de Psicología de la Educación, aunque hay muchas definiciones para esta palabra para mi aquella que engloba todo es que la ciencia es "un conjunto de conocimientos racionales, ciertos o probables, obtenidos metódicamente, sistematizados, verificables, que hacen referencia a un ámbito específico de la realidad. El conocimiento es acumulativo y la teoría

Ciclos de Mejora en el Aula (2020). Experiencias de Innovación Docente de la US Esta obra se distribuye con la licencia Creative Commons 
generaliza hacia conjuntos de categorías similares (A. 25).

Pregunta 1. Me gustaría que definieses con tus palabras ¿qué es ciencia?

Categoría 1: Grado de reconocimiento del concepto de ciencia. Problema 1, subproblema 1 (E. Natural).

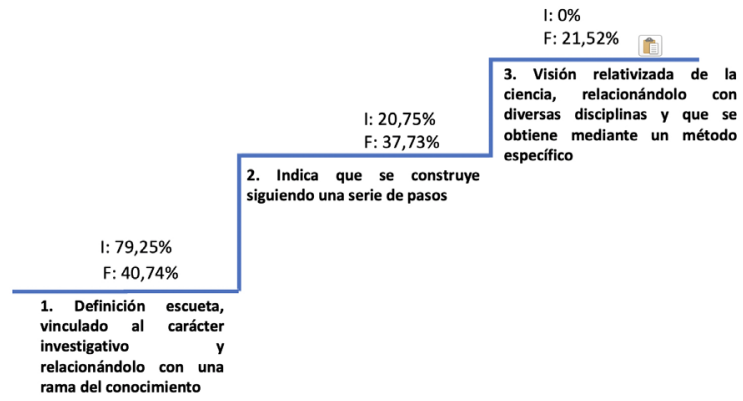

Figura 3. Escalera de aprendizaje Pregunta 1

En la figura 4, podemos observar que se produce una evolución significativa que lleva a que un $62 \%$ del alumnado llegue al nivel 4, en el que identifican el conocimiento científico con el método científico y son capaces de describir el proceso de investigación de modo coherente, partiendo de una pregunta-problema, enumerando los diferentes pasos a seguir. Destacamos también que, mientras al inicio el 59\% del alumnado se encontraba en el nivel 2 , al final del bloque, solo un $10 \%$ se sitúa en este nivel, lo que nos hace ver que han logrado superar la visión más simple para llegar a una más compleja sobre el proceso de investigación.

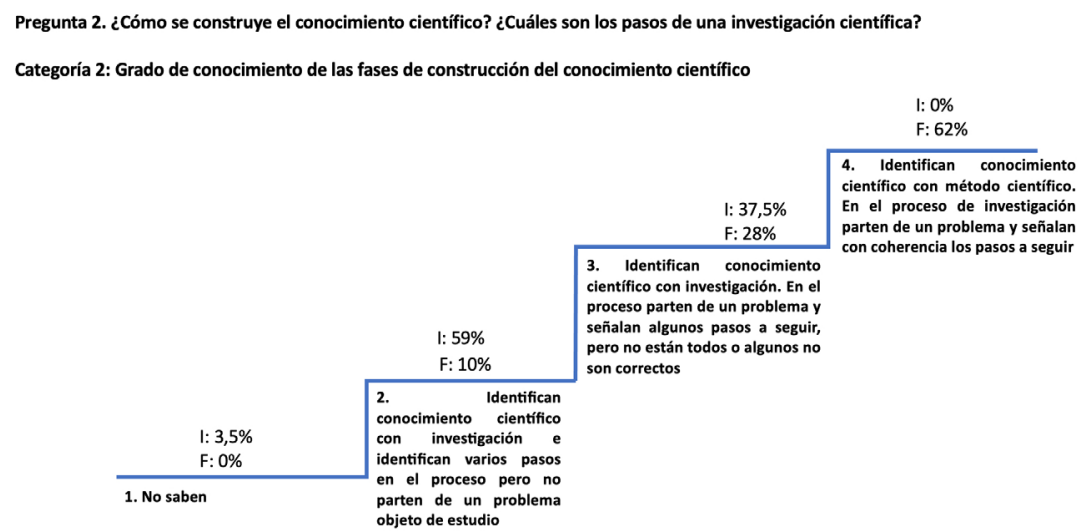

Figura 4. Escalera de aprendizaje Pregunta 2

Ciclos de Mejora en el Aula (2020). Experiencias de Innovación Docente de la US Esta obra se distribuye con la licencia Creative Commons 
Para el análisis de la pregunta 3, representada en la figura 5, se han subdividido las respuestas en dos subcategorías según hiciesen alusión al ámbito del problema (local, global o ambos) o al carácter social, ambiental o socioambiental del problema. En este sentido, en la primera subcategoría hay que resaltar que el alumnado percibe problemas globales con mayor facilidad que los locales. Como ejemplo, podemos indicar que varios alumnos (A.3, A.24, A.26,...) consideran el incendio del Amazonas del año 2019 frente a una sola persona que enumera el problema de los incendios en el ámbito local: "son muy comunes en nuestra región y afectan gravemente a la biodiversidad de España, además de provocar daños graves en las personas que viven cercanas a ellos" (A. 31). En relación con la subcategoría 3b, antes de la intervención se percibían problemas de carácter más ambiental como la contaminación frente a los socioambientales, no habiendo ninguna persona que indicase problemas de carácter social únicamente. Al final de la intervención se observa un avance en las respuestas que indican problemas socioambientales, con un $68 \%$ de las respuestas, intuyendo que con la intervención han reconocido que los problemas es difícil analizarlos desde una única perspectiva. Algunos de los problemas más nombrados en este caso, han sido el Cambio climático y la contaminación derivada de la industria textil.

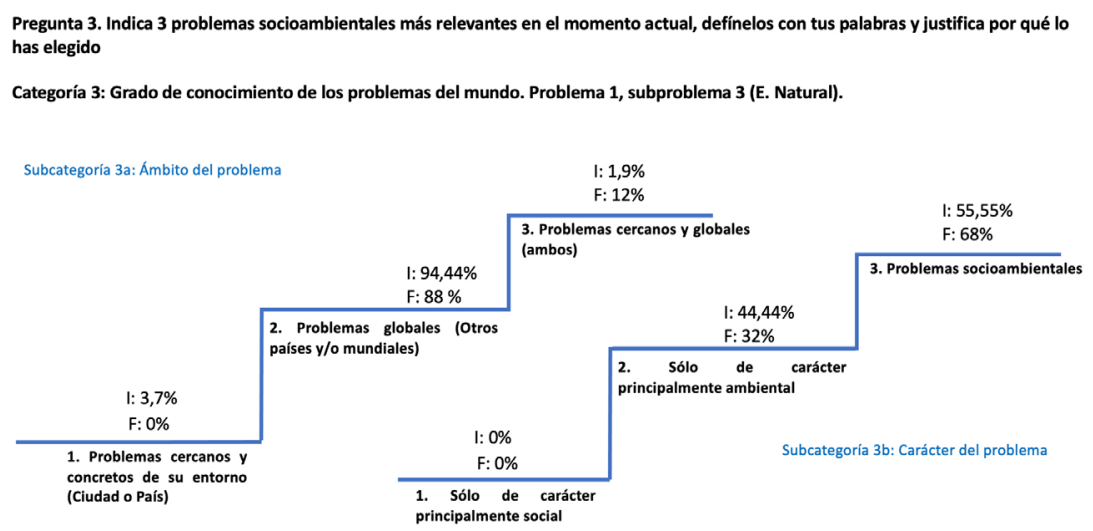

Figura 5. Escalera de aprendizaje Pregunta 3

Ciclos de Mejora en el Aula (2020). Experiencias de Innovación Docente de la US Esta obra se distribuye con la licencia Creative Commons 
En la última pregunta de nuestro cuestionario (figura 6), también apreciamos evolución en los conocimientos del alumnado, donde de un 55,3\% de respuestas simples, sin argumentación, pasamos a un $46 \%$ de respuestas complejas, que incluyen reflexión sobre la relación entre la situación actual del mundo y la educación. Sin embargo, observamos que sigue existiendo un porcentaje elevado de estudiantes (44\%) que tras el desarrollo del bloque 1 , manifiesta una respuesta de complejidad media, quedándose en ejemplos algo simple y sin profundidad en sus argumentos. Por tanto, consideramos que estos datos nos indican que se hace necesario seguir trabajando en este aspecto a lo largo de la asignatura.

\footnotetext{
Pregunta 4 . La ciencia y la situación actual de nuestro mundo afecta a la educación que tenemos que dar a nuestros niños y niñas ¿En
} qué? Pon algún ejemplo concreto.

Categoría 4: Relación ciencia, situación del mundo y educación. Problema 1, subproblema 3 (E. Natural) y subproblema 3 (E. Social).

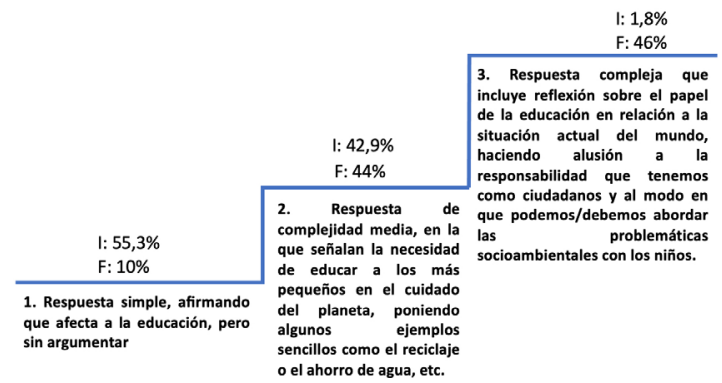

Figura 6. Escalera de aprendizaje Pregunta 4

\section{Evaluación del CIMA}

A pesar de no haber podido obtener aún datos relativos al desarrollo completo de ambas materias, los resultados que arrojan los primeros análisis de los cuestionarios de los alumnos, nos hacen valorar de manera positiva la experiencia. Sin duda, este curso está siendo especialmente complejo y las dificultades derivadas de las modalidades de enseñanza (primero semipresencial y después virtual) a las que hemos tenido que irnos adaptando, nos han hecho

Ciclos de Mejora en el Aula (2020). Experiencias de Innovación Docente de la US Esta obra se distribuye con la licencia Creative Commons 
tener que dar muchas vueltas al modo en que abordábamos cada una de las sesiones, para garantizar la máxima participación del alumnado. En todo momento, hemos intentado poner al alumnado en el centro del proceso y que fuera parte activa del proceso de aprendizaje. La visión que cada una de las dos docentes íbamos teniendo respecto al desarrollo del CIMA, nos ha servido para descubrir puntos de vista diferentes y en muchas ocasiones complementarios. Por eso, a pesar de que la coordinación en este curso tan peculiar supone un sobresfuerzo, creemos que está mereciendo la pena.

Uno de los aspectos sobre los que debemos de seguir avanzando es sobre la necesidad de ajuste del tiempo. Generalmente, cuando planificamos, caemos en el error de considerar que en una sesión de clase se pueden realizar diversas actividades, pero este curso especialmente, estamos comprobando que las sesiones de clase quedan bastante reducidas y que necesitamos quedarnos con aquellas actividades que consideramos esenciales. En estrecha relación con esto, creemos que en lo que queda de cuatrimestre, tendremos que eliminar algunos de los contenidos del bloque 2, puesto que nos va a resultar imposible desarrollarlo por completo. Así, tenemos previsto reducir el problema 2 "Ideas de los alumnos" junto con los subproblemas 1 y 2 "¿Qué saben los niños y niñas de Infantil sobre ciencias? ¿Cómo son sus ideas? ¿Cómo podemos averiguarlo? ¿Para qué nos sirve?". Dada la situación actual y debido al desajuste de tiempo, suprimiremos la actividad en la que el alumnado, una vez diseñado el instrumento de detección de ideas lo administraba a niños y niñas de Educación Infantil para posteriormente analizarlo. En su lugar, proporcionaremos las docentes ejemplos ya transcritos de conversaciones con los niños y se realizarán en clase a modo de ejemplos. Esto, aunque más alejado de la práctica real, nos permitirá abordar los contenidos claves de este problema en menor tiempo. En este sentido, debido a que la interacción entre el alumnado se está viendo

Ciclos de Mejora en el Aula (2020). Experiencias de Innovación Docente de la US Esta obra se distribuye con la licencia Creative Commons 
mermado, que el trabajo práctico y vinculado a la realidad también, aspectos que impiden la comprensión de contenidos complejos que abordamos, consideramos necesario introducir actividades de trabajo cooperativo online que favorezcan estas interacciones y sustituir las actividades prácticas de clase por otras más accesibles.

Finalmente, en lo que respecta al modelo didáctico seguido, consideramos que, aunque siempre es necesario seguir avanzando, nos va funcionando bien y nos permite seguir trabajando, acercándonos a nuestro modelo didáctico ideal (Porlán, 2018), gracias en gran parte a la coordinación docente por la que venimos apostando.

Ciclos de Mejora en el Aula (2020). Experiencias de Innovación Docente de la US Esta obra se distribuye con la licencia Creative Commons 
Palabras claves: Enseñanza del entorno natural, conocimiento del entorno social, educación infantil, docencia universitaria, experimentación docente universitaria, coordinación docente.

Keywords: Teaching of the natural environment, knowledge of the social environment, early child hood education, university education, university teaching experimentation, to teaching coordination.

\section{Referencias}

Porlán, R. (Coord.) (2017). Enseñanza universitaria. Cómo mejorarla. Madrid: Morata

Puig, M. (2018). La enseñanza del entorno a través de rincones. En R. Porlán y E. Navarro-Medina (Coord.), Jornadas de Formación Docente e Innovación Docente (pp.1855-1873). Sevilla: Editorial Universidad de Sevilla. DOI: http://dx.doi.org/10.12795/JDU.2018.101.104

Rodríguez-Marín, F. (2018). ¿Son los rincones una buena estrategia metodológica para la enseñanza del Entorno en Educación Infantil? En R. Porlán y E. Navarro-Medina (Coord.), Jornadas de Formación Docente e Innovación Docente (pp.1741-1755). Sevilla: Editorial Universidad de Sevilla. DOl: http://dx.doi.org/10.12795/JDU.2018.i01.98

Ciclos de Mejora en el Aula (2020). Experiencias de Innovación Docente de la US Esta obra se distribuye con la licencia Creative Commons 